\title{
ON THE LOCATION OF THE ZEROS OF THE DERIVATIVE OF A POLYNOMIAL
}

\author{
J. BRIAN CONREY AND LEE A. RUBEL ${ }^{1}$
}

\begin{abstract}
We show that nonreal zeros of a real polynomial $P(z)$ sometimes induce nonreal zeros of $P^{\prime}(z)$.
\end{abstract}

1. Introduction. We prove two results in the geometry of the zeros of polynomials [2]. In particular, we study how the distribution of the zeros of a real polynomial may force (or prevent) the existence of a nonreal zero of its derivative. Our investigation was originally motivated by certain considerations about the zeros of Riemann's xi-function (and of its derivative) which arise in analytic number theory (see [1]). We note that our method applies also to real entire functions of order $\leq 1$.

2. Notation. For simplicity we will work with polynomials though the proofs are valid in a more general situation. We assume henceforth that $P$ is a polynomial with real coefficients. Generic real zeros of $P$ will be denoted $r$ and complex conjugate pairs are $w$ and $\bar{w}$ where $w=u+i v$. (To indicate specific zeros we use subscripts.) We associate to $w$ the Jensen disc

$$
J_{w}=\{z:|z-u| \leq v\} .
$$

A well-known theorem of Jensen [2] asserts that if $P^{\prime}(z)=0$ then either $z$ is real or $z \in J_{w}$ for some $w$. This follows immediately from the fact that if $f(z)=$ $(z-w)(z-\bar{w})=(z-u)^{2}+v^{2}$, then

$$
\operatorname{Im} \frac{f^{\prime}}{f}(z)=-2 y \frac{|z-u|^{2}-v^{2}}{\left|(z-u)^{2}+v^{2}\right|^{2}}
$$

(so that $\operatorname{Im} P^{\prime}(z) / P(z) \neq 0$ if $z$ is nonreal and outside every Jensen disc).

We also write $z=x+i y$.

3. Statement of results. Our first theorem asserts that if a real polynomial $P$ has real zeros "near" to the projection of a complex zero $w_{0}$ of $P$ onto the real axis, then $P^{\prime}$ has a nonreal zero, provided that $w_{0}$ is, in a certain sense, isolated. By "near" we mean within a distance of the order of magnitude of $\left|\operatorname{Im} w_{0}\right|$.

THEOREM 1. Let $n \geq 1$ be fixed and let

$$
c_{n}= \begin{cases}0.5 & \text { if } n=1 \\ \frac{1}{2}\left(\sqrt{9 n^{2}-8 n}-n\right)^{1 / 2} & \text { if } n>1\end{cases}
$$

Received by the editors December 15, 1981. The contents of this paper have been presented at the Austin Meeting of the AMS on November 7, 1981 at the special session on number theory and related parts of analysis.

1980 Mathematics Subject Classification. Primary 30C15.

${ }^{1}$ The research of the second author was partially supported by a grant from the National Science Foundation. 
Suppose that $P\left(w_{0}\right)=P\left(r_{1}\right)=\cdots=P\left(r_{n}\right)=0$ and that $\left|u_{0}-r_{i}\right|<c_{n}\left|v_{0}\right|$ for $1 \leq i \leq n$. Suppose further that $J_{w} \cap J_{w_{0}}=\emptyset$ where $w$ ranges over the nonreal zeros of $P$ with $w \neq w_{0}, \bar{w}_{0}$. Then $P^{\prime}$ has a nonreal zero inside $J_{w_{0}}$.

We can obtain a more precise result if the zeros of $P$ are symmetric about the $y$-axis.

THEOREM 2. Suppose that $P$ is an even function, $P(0) \neq 0$, and $P\left(i v_{0}\right)=0$ where $v_{0}>0$. Let $r_{0}$ be the smallest real zero of $P$ such that $r_{0} \geq v_{0}$. Suppose that $J_{w} \cap\left[-r_{0}, r_{0}\right]=\emptyset$ where $w$ ranges over the nonreal zeros of $\bar{P}$ with $w \neq \pm i v_{0}$. Define

$$
K=\sum_{r} \frac{1}{r^{2}}+\sum_{w} \frac{u^{2}-v^{2}}{\left(u^{2}+v^{2}\right)^{2}}
$$

Then $P^{\prime}$ has a nonreal zero inside $J_{i v_{0}}$ if and only if $K>0$.

It follows from this theorem that $c_{n}$ of Theorem 1 is asymptotically best possible. For if the zeros of $P$ consist of $n$ (where $n$ is even) real zeros all at $\pm r_{0}$ and a pair of complex zeros at $\pm i v_{0}$ then Theorem 2 asserts that $P^{\prime}$ has only real zeros if

$$
K=\frac{n}{r_{0}^{2}}-\frac{2}{v_{0}^{2}}<0,
$$

that is, if $r_{0}>\sqrt{n / 2} v_{0}$; Theorem 1 asserts that $P^{\prime}$ has a nonreal zero if $r_{0}<c_{n} v_{0}$. Since $c_{n} \sim \sqrt{n / 2}$ as $n \rightarrow \infty$, Theorem 1 is nearly sharp.

4. Proofs. We now prove Theorem 1. We may assume without loss of generality that $u_{0}=0$ so that $w_{0}=i v_{0}, v_{0}>0$. Then

$$
P(z)=\left(z^{2}+v_{0}^{2}\right)\left(z-r_{1}\right) \cdots\left(z-r_{n}\right) Q(z)
$$

where $Q$ is a polynomial with real coefficients. Consider the contour

$$
\begin{aligned}
C_{\epsilon} & =\left\{z:|z-i \epsilon|=v_{0} \text { and } y>\epsilon\right\} \cup\left\{z=x+i \epsilon:-v_{0} \leq x \leq v_{0}\right\} \\
& =C_{\epsilon}^{U}+C_{\epsilon}^{L}
\end{aligned}
$$

where $\epsilon$ is a small positive number. We will show that if $\epsilon$ is small enough then $\operatorname{Im} P^{\prime}(z) / P(z)<0$ on $C_{\epsilon}$. Then the change in the argument of $P^{\prime}(z) / P(z)$ as $z$ varies around $C_{\epsilon}$ is 0 . Hence by the argument principle the number of zeros of $P^{\prime}$ inside $C_{\epsilon}$ is equal to the number of zeros of $P$ inside $C_{\epsilon}$, namely one $\left(P\left(i v_{0}\right)=0\right)$, which shows the result.

By an easy calculation, $\operatorname{Im} P^{\prime}(z) / P(z)=-y F(z)+\operatorname{Im} Q^{\prime}(z) / Q(z)$ where

$$
F(z)=2 \frac{|z|^{2}-v_{0}^{2}}{\left|z^{2}+v_{0}^{2}\right|^{2}}+\sum_{i=1}^{n} \frac{1}{\left|z-r_{i}\right|^{2}}
$$

By the hypothesis about the Jensen discs of $P, \operatorname{Im} Q^{\prime}(z) / Q(z)<0$ for $z$ on $C_{\epsilon}$, if $\epsilon$ is small enough. Also, $F(z)>0$ for $z$ on $C_{\epsilon}^{U}$ for any $\epsilon>0$. Finally we will show that $F(z)>0$ for $z$ on $C_{0}^{L}$ which implies that $F(z)>0$ for $z$ on $C_{\epsilon}^{L}$, if $\epsilon>0$ is sufficiently small. Hence we must show that

$$
F(x)=F\left(x, r_{1}, r_{2}, \ldots, r_{n}\right)=2 \frac{x^{2}-v_{0}^{2}}{\left(x^{2}+v_{0}^{2}\right)^{2}}+\sum_{i=1}^{n} \frac{1}{\left(x-r_{i}\right)^{2}}>0
$$


for $|x| \leq v_{0}$ and $\left|r_{i}\right| \leq c_{n} v_{0}$. We consider the minimum $M$ of $F$ on this set. By symmetry, a minimum value of $F$ will occur in $x \geq 0$. Since $\partial F / \partial r_{i}$ is never 0 , the minimum for $F$ occurs when each $r_{i}= \pm c_{n} v_{0}$. Therefore,

$$
M=\min _{0 \leq x \leq v_{0}} 2 \frac{x^{2}-v_{0}^{2}}{\left(x^{2}+v_{0}^{2}\right)^{2}}+\frac{n}{\left(x+c_{n} v_{0}\right)^{2}} .
$$

Let $f(x)=2\left(x^{2}-v_{0}^{2}\right)\left(x+c_{n} v_{0}\right)^{2}+n\left(x^{2}+v_{0}^{2}\right)^{2}$. Then to prove $M>0$ it is sufficient to show that $f(x)>0$ for $0 \leq x \leq v_{0}$ or, equivalently, that

$$
g(x)=2\left(x^{2}-1\right)\left(x+c_{n}\right)^{2}+n\left(x^{2}+1\right)^{2}>0
$$

for $0 \leq x \leq 1$. For $n=1$ and $c_{1}=0.5$ this is easily shown by Sturm's Theorem (see [3]). For $n>1$ we observe that

$$
\begin{aligned}
g(x) & =(n+2) x^{4}+4 c_{n} x^{3}+2\left(n-1+c_{n}^{2}\right) x^{2}-4 c_{n} x+\left(n-2 c_{n}^{2}\right) \\
& \geq 2\left(n-1+c_{n}^{2}\right) x^{2}-4 c_{n} x+\left(n-2 c_{n}^{2}\right)
\end{aligned}
$$

for $x \geq 0$ if $c_{n} \geq 0$. The last expression in positive for all $x$ if

$$
0>16 c_{n}^{2}-8\left(n-1+c_{n}^{2}\right)\left(n-2 c_{n}^{2}\right)=8\left(2 c_{n}^{4}+n c_{n}^{2}-n(n-1)\right)
$$

which holds if $c_{n}^{2} \leq\left(-n+\sqrt{n^{2}+8 n(n-1)}\right) / 4$.

To prove Theorem 2 we introduce an auxiliary polynomial

$$
P_{t}(z)=\left(z^{2}+t^{2} v_{0}^{2}\right) \prod_{r}(z-r) \prod_{\substack{w \neq i v_{0} \\ v>0}}\left((z-u)^{2}+v^{2}\right)
$$

so that $P_{t}$ and $P$ have the same zeros except that $P$ is zero at $\pm i v_{0}$ while $P_{t}$ is zero at $\pm i t v_{0}$. Let $C_{\epsilon}$ be the circle of radius $r_{0}+\epsilon$ with center at the origin. Let $\epsilon>0$ be so small that $\left[-r_{0}-\epsilon, r_{0}+\epsilon\right] \cap J_{w}=\emptyset$ for all $w \neq \pm i v_{0}$. Then (as in Jensen's Theorem) $\operatorname{Im} P_{t}^{\prime}(z) / P_{t}(z)$ is zero only at the two real points of $C_{\epsilon}$, for $0 \leq t \leq 1$. Therefore the change in $\arg P_{t}^{\prime}(z) / P_{t}(z)$ on $C_{\epsilon}$ is 0 or $\pm 2 \pi$ so that, by the argument principle, the number of zeros of $P_{t}^{\prime}(z)$ inside $C_{\epsilon}$ differs from the number of zeros of $P_{t}(z)$ inside $C_{\epsilon}$ by at most one, for $0 \leq t \leq 1$. By Rolle's Theorem, $P_{t}^{\prime}(x)$ has an odd number of real zeros strictly between consecutive real zeros of $P_{t}(x)$. To help envision the proof, imagine that we are watching a movie of the zeros of $P_{t}^{\prime}$ as $t$ moves from 0 to 1 . When $t=0, P_{t}(z)=P_{0}(z)$ has only real zeros inside or on $C_{0}$ (the circle of radius $r_{0}$ with center the origin). Moreover, by Jensen's Theorem, $P_{0}^{\prime}(z)$ has only real zeros inside $C_{0}$, and by the above remarks $P_{0}^{\prime}(x)$ has precisely one real zero (counting multiplicities) between consecutive real zeros of $P_{0}(x)$, for $-r_{0} \leq x \leq r_{0}$. For if $P_{0}^{\prime}(x)$ had three (or more) zeros between consecutive real zeros $r$ and $r^{\prime}$ of $P_{0}(x)$ then (since $P_{0}^{\prime}$ is an odd function) $P_{0}^{\prime}$ would have three or more zeros between $-r^{\prime}$ and $-r$ and the total number of zeros of $P_{0}^{\prime}$ in $\left[-r_{0}, r_{0}\right]$ would exceed that of $P_{0}$ by at least three, in contradiction to what we deduced from the argument principle. Thus, when the movie starts the zeros of $P_{t}^{\prime}$ inside $C_{0}$ are real and simple (except if $P_{0}$ has a zero of multiplicity $m \geq 3$ at some $r \neq 0$ which causes a zero of $P_{0}^{\prime}$ of multiplicity $m-1$ at $r$ ) and interlace the zeros of $P_{0}$ (all of which are real). Also $P_{0}$ has a double zero at the origin.

Now the zeros of $P_{t}^{\prime}$ are continuous functions of $t$. Therefore no new nonreal zeros of $P_{t}^{\prime}$ can enter $C_{0}$ as $t$ varies from 0 to 1 , for nonreal zeros of $P_{t}^{\prime}$ must remain in the various Jensen discs, none of which intersects $C_{0}$ (except, of course, $J_{ \pm i v_{0}}$ ). 
Also, a real zero of $P_{t}^{\prime}$ cannot coalesce with a (previously distinct) real zero of $P$. For if $r \neq 0$ is a zero of $P$ of multiplicity $m>0$ then $r$ is a zero of $P_{t}$ of multiplicity $m$ and therefore a zero of $P_{t}^{\prime}$ of multiplicity (precisely) $m-1$. Thus, as the movie progresses $(0 \leq t \leq 1)$ no new zeros of $P_{t}^{\prime}$ enter or leave $C_{0}$ and the real zeros of $P_{t}^{\prime}$ which are between the consecutive zeros $r, r^{\prime}$ of $P$ with $r<r^{\prime}<0$ (or $r>r^{\prime}>0$ ) must remain real and between $r$ and $r^{\prime}$.

Let $0<r_{1} \leq r_{0}$ with $P\left(r_{1}\right)=0$ and $P(x) \neq 0$ for $-r_{1}<x<r_{1}$. The zeros of $P_{t}^{\prime}$ in $\left(-r_{1}, r_{1}\right)$ will behave differently from those described above. When $t=0$, there are three zeros of $P_{t}^{\prime}$ in $\left(-r_{1}, r_{1}\right)$ : one in $\left(-r_{1}, 0\right)$, one in $\left(0, r_{1}\right)$ and one at the origin. The one at the origin remains there for all $t$. The other two will vary with $t$ : call them $S_{t}^{ \pm}$, with $S_{0}^{+}>0$. It is clear from what has been so far described that the only way for $P^{\prime}(z)$ to have nonreal zeros inside $C_{0}$ is if $S_{t}^{+}$and $S_{t}^{-}$coalesce at the origin and become (and remain) purely imaginary conjugates. We will now show that if $K \leq 0$ then $S_{1}^{ \pm}$are real while if $K>0$ then $S_{1}^{ \pm}$are purely imaginary nonreals.

If $S_{1}^{ \pm}$are nonreal then for some $t_{0}, 0<t_{0}<1, P_{t_{0}}^{\prime}$ has a triple zero at the origin. Hence if $P_{t}^{\prime \prime}(0) \neq 0$ for $0<t<1$ then $S_{1}^{ \pm}$are real. But an easy calculation gives

$$
P_{t}^{\prime \prime}(x)=P_{t}^{\prime}(x) \cdot \frac{P_{t}^{\prime}}{P_{t}}(x)+P_{t}(x) \frac{d}{d x}\left(\frac{P_{t}^{\prime}}{P_{t}}(x)\right)
$$

so that $P_{t}^{\prime \prime}(0)=-P_{t}(0) K(t)$ (since $\left.P_{t}^{\prime}(0)=0\right)$ where

$$
K(t)=\frac{2}{t^{2} v_{0}^{2}}+\sum_{r} \frac{1}{r^{2}}+\sum_{w \neq \pm i v_{0}} \frac{u^{2}-v^{2}}{\left(u^{2}+v^{2}\right)^{2}} .
$$

Since $P_{t}(0) \neq 0$ it follows that $K(t) \neq 0$ for $0<t<1$ implies that $S_{1}^{ \pm}$are real. Clearly $K$ is an increasing function of $t$ which is negative for all sufficiently small $t>0$. Therefore, if $K=K(1)<0$ then $K(t)<0$ for all $t, 0<t<1$. Hence $S_{1}^{ \pm}$ are real in the event that $K<0$, as was asserted in the theorem.

To prove the other assertion we consider

$$
P_{\infty}(z)=\prod_{r}(z-r) \prod_{\substack{w \neq \pm=i v 0 \\ v>0}}\left((z-u)^{2}+v^{2}\right),
$$

so that $P_{\infty}(z)=P(z) /\left(z^{2}+v^{2}\right)$. With the same argument we used above we conclude that within $C_{\epsilon}$ the zeros of $P_{\infty}^{\prime}$ are all real and interlaced with the zeros of $P_{\infty}$. In particular, if $-r_{1}<x<r_{1}$ then $P_{\infty}^{\prime}(x)=0$ only when $x=0$. Now if $t$ is sufficiently large (we no longer require $t \leq 1$ ), then $P_{t}^{\prime}$ and $P_{\infty}^{\prime}$ will have the same number of zeros in $-r_{1}<x<r_{1}$. This is because

$$
\left|\frac{P_{\infty}^{\prime}}{P_{\infty}}(z)-\frac{P_{t}^{\prime}}{P_{t}}(z)\right|=\left|\frac{2 z}{z^{2}+t^{2} v_{0}^{2}}\right|
$$

so that $P_{t}^{\prime}(x) / P_{t}(x)$ is bounded away from 0 when $P_{\infty}^{\prime}(x) / P_{\infty}(x)$ is (for large $t$ ) while in a small neighborhood of the origin (which is the only zero of $P_{\infty}^{\prime}(x) / P_{\infty}(x)$ in $\left.-r_{1}<\dot{x}<r_{1}\right) P_{t}^{\prime} / P_{t}$ and $P_{\infty}^{\prime} / P_{\infty}$ have the same number of zeros by Rouché's Theorem. Therefore, for all sufficiently large $t$, the zeros $S_{t}^{ \pm}$ere not real. Nonreal zeros of $P_{t}^{\prime}$ which are not purely imaginary occur in sets of four, $( \pm w, \pm \bar{w})$, since $P_{t}^{\prime}$ is an odd, real function. Therefore, as soon as $t$ is large enough so that $S_{t}^{ \pm}$are no longer real they must be purely imaginary and the transition occurs at some $t_{0}$ 
with $-P_{t_{0}}(0) K\left(t_{0}\right)=P_{t_{0}}^{\prime \prime}(0)=0$. But if $K(1)=K>0$ then $K(t)>0$ for all $t \geq 1$, since $K(t)$ is an increasing function. Therefore, if $K(1)>0$ then there is a $t_{0}<1$ such that $K\left(t_{0}\right)=0$ and $S_{t}^{ \pm}$are nonreal for all $t>t_{0}$. In particular $S_{1}^{ \pm}$ are nonreal and are inside $J_{i v_{0}}$, by Jensen's Theorem. This proves Theorem 2.

5. An open question. We wonder to what extent the hypothesis in Theorem 1, $J_{w} \cap J_{w_{0}}=\emptyset$, is needed. For the application we have in mind we would like to be able to remove this hypothesis in the case that all the zeros of $P(z)$ are in a strip

$$
-c_{0}<\operatorname{Im}\{z\}<c_{0}
$$

and $\left|v_{0}\right|>\frac{1}{2} c_{0}$. We pose the question whether or not a similar conclusion to Theorem 1 can be drawn in this case.

\section{REFERENCES}

1. B. Conrey, Zeros of derivatives of Riemann's xi-function on the critical line, J. Number Theory (to appear).

2. M. Marden, The geometry of the zeros of a polynomial in a complex variable, Amer. Math. Soc., Providence, R. I., 1949.

3. H. S. Wilf, Mathematics for the physical sciences, Wiley, New York, 1962.

DEPARTMENT OF MATHEMATICS, UNIVERSITY OF ILliNOIS, URBANA, ILLINOIS 61801 